\title{
Correction to: Human Cytomegalovirus Influences Host circRNA Transcriptions during Productive Infection
}

\author{
Jingui Deng ${ }^{1,2} \cdot$ Yujing Huang $^{1}\left(\mathbb{D} \cdot\right.$ Qing Wang $^{1} \cdot$ Jianming $\mathrm{Li}^{1} \cdot$ Yanping Ma ${ }^{1} \cdot$ Ying $\mathrm{Qi}^{1} \cdot \mathrm{Zhongyang}^{\mathrm{Liu}}{ }^{1} \cdot$ \\ Yibo $\mathrm{Li}^{3,4} \cdot$ Qiang Ruan ${ }^{1}$ (D)
}

Published online: 10 November 2020

(C) Wuhan Institute of Virology, CAS 2020

\section{Correction to: Virologica Sinica}

https://doi.org/10.1007/s12250-020-00275-6

The information of affiliation 1 and 3 was incorrect. The information of affiliations should read as given below.

Author information

1 Virology Lab, Shengjing Hospital of China Medical University, Shenyang 110004, China

2 Department of Laboratory, Central Hospital Affiliated to Shenyang Medical College, Shenyang 110024, China

The original article can be found online at https:// doi.org/10.1007/s12250-020-00275-6.

Qiang Ruan

ruanq@sj-hospital.org

$\bowtie$ Yujing Huang

huangyj@sj-hospital.org

1 Virology Lab, Shengjing Hospital of China Medical University, Shenyang 110004, China

2 Department of Laboratory, Central Hospital Affiliated to Shenyang Medical College, Shenyang 110024, China

3 Department of Obstetrics and Gynecology, Shengjing Hospital of China Medical University, Shenyang 110004, China

4 Department of Obstetrics, Central Hospital Affiliated to Shenyang Medical College, Shenyang 110024, China 